\title{
Ant Based Cross Layered Optimization Protocol for WMSN with Fuzzy Clustering
}

\author{
DipaliParag Adhyapak ${ }^{1}$, Sridharan Bhavani ${ }^{2}$, Aparna Pradeep Laturkar ${ }^{3}$ \\ ${ }^{1,3}$ Department of Electronics \& Telecommunication, PES's Modern College of Engineering, Savitribai Phule Pune \\ University, Pune, India \\ ${ }^{2}$ Department of Electronics \& Communication, Karpagam Academy of Higher Education, Coimbtore, India
}

\begin{tabular}{|c|c|}
\hline Article Info & ABSTRACT \\
\hline & Wireless Multimedia Sensor Network (WMSN) is embedded with large \\
\hline Received Dec 9, 2017 & the multimedia information from the environment. WMSN has several \\
\hline Revised Feb 28, 2018 & challenges such as life time of the network, Memory requirement, Coverage, \\
\hline Accepted Mar 18, 2018 & $\begin{array}{l}\text { Bandwidth and QoS metrics. Hence selection of routing algorithm is crucial } \\
\text { in WMSN. Again interdependencies of the protocol layer cannot be }\end{array}$ \\
\hline Keywords: & $\begin{array}{l}\text { neglected to improve the network performance. Clustering in WMSN is } \\
\text { challenging task in order to increase network lifetime and to improve the }\end{array}$ \\
\hline WMSN & communication. Hence Fuzzy clustered Ant based cross layer protocol \\
\hline QoS & $\begin{array}{l}\text { (FCAXL) is proposed. In this paper performance analysis of ant based cross } \\
\text { layer optimization protocol with fuzzy clustering based on number of nodes }\end{array}$ \\
\hline FCAXL & $\begin{array}{l}\text { and packet size is done. Simulation results shows that Fuzzy clustered ant } \\
\text { based cross layer optimization protocol performs best as compared to } \\
\text { AntSenseNet routing protocol, Cross layer routing protocol and Ant based } \\
\text { cross layer routing protocol in terms of QoS parameters such as Throughput, } \\
\text { Packet delivery ratio and delay. Hence the life time of the network increases. }\end{array}$ \\
\hline
\end{tabular}

All rights reserved.

\section{Corresponding Author:}

DipaliParag Adhyapak,

Karpagam University,

Pollachi Main Road, L \& T Bypass road Junction,

Eachanari Post, Eachanari, Coimbatore, Tamilnadu, India- 641021.

Email: adhyapakdeepa@gmail.com

\section{INTRODUCTION}

Wireless sensor networks are comprised of large number of scalar sensor nodes which can sense physical parameters like temperature, pressure, humidity, sound and light and can exchange the information. Advancement in technology has led to the multimedia sensor nodes which are capable of sensing audio as well as video data. These multimedia devices are embedded in the sensor node. Such type of networks is called as wireless multimedia sensor networks. These networks can sense and transfer the scalar as well as multimedia data i. e. image, audio, and video streams in real time as well as non-real time communication. These networks have additional features like high bandwidth requirement, tolerable delay, low jitter, low packet loss which imposes the additional challenges on the designer. WMSN is in the need of on time reliable data delivery to achieve Quality of Service. Multimedia data is delay sensitive [1] and sensitive to packet loss also which may result in jitter and decreases the throughput.

Selection of routing protocol plays an important role for WMSN networks. Main objective of the routing protocol is to minimize the end to end delay, jitter and to increase the packet delivery ratio in turn to increase the throughput atlow energy cost. These networks are generally event based networks. For event based networks proactive approach is used in which each node monitors its neighboring links and update changes accordingly [1]. Sensor nodescommunicate with each other to detect events depending on the application, to collect and process data, and to transmit the sensed information to the base station by hopping 
the data from node to node [7]. The senor nodes are either randomly deployed or according to the statistical distribution. Wireless mulitimedia sensor network consist of various sensor nodes that are used to target or monitor forest area or industrial activity. [8].

Swarm based routing protocols have been used to tackle additional challenges. Most successful and famous swarm intelligence is the ant colony optimization technique [2]. In this, artificial ants are used to find the most efficient path. Also interdependencies of the protocol layer cannot be neglected. To exploit these interdependencies cross layering principle can be employed along with the swarm intelligence. These WMSN networks are having hierarchical structure. Hence to optimize the clustering process, fuzzy is the one of the best solution. Fuzzy based clustering minimizes dead nodes, saves the cost of creating new clusters, increases the residual energy and in turn enhances the network lifetime.

In this paper Fuzzy clustered ant based cross layer protocol is proposed for WMSN which minimizes end to end delay, increases packet delivery ratio and throughput. Ant based routing protocol is used to find the shortest path while the priority based scheduling and queuing minimizes the delay. Fuzzy based clustering minimizes cost of creating new clusters which in turn enhances the network lifetime.

The Network simulator helps the developer to create and simulate new models on an arbitrary network byspecifying both the behavior of the network nodes and the communication channels. It provides a virtualenvironment for an assortment of desirable features such as modeling a network based on a specific criteria andanalyzing its performance under different scenarios [9]. Hence to simulate the proposed algorithm Network Simulator 2 (NS2) is used.

The remainder of this paper is organized as follows. In section II some related work is discussed. In section III, FCAXL-Routing protocol is discussed. Section IV discusses about the performance of the proposed protocol.

\section{LITERATURE SURVEY}

In this section a brief survey of WMSN and its routing is presented. Wireless multimedia sensor network has many challenges.Several approaches have been proposed to optimize the QoS parameters. Cross layer QoS routing protocol for WMSN has been proposed by Zara Hamid, Faisal Bashir, Jae Young Pyun. This protocol has aimed at providing soft end to end delay guarantees. Evaluation has showed that it provides better delay as compared to minimum routing protocol [1].

Luis Cobo, Alejandro and Samuel has proposed QoS routing model based on traditional ant based algorithm. AntSenseNet protocol has been implemented. This algorithm has better convergence and it provides significantly better QoS for multiple types of services [2].

Cross layer ant based routing protocol for WMSN has been proposed by M. Abazeed, K. Saleem, S. Zubair and N. Fisal in 2011. He has used modified ACO technique to enhance the routing efficiency. This improved ACO has been used to search for the best path to satisfy the multimedia traffic requirements [3].

Fuzzy based approach of energy efficient hierarchical clustering is proposed by G. S. M. Vamsi and NehaChoubey. They have optimized the clustering process, cluster head election, and decreased the number of dead nodes. Cluster selection is random in nature and weights have been calculated based on which cluster head election is done. Hierarchical routing has been implemented using fuzzy interference engine. By applying this method the residual energy and network lifetime has been increased whereas the cost of creating the clusters and number of dead nodes has been decreased [4].

VikasBhandarary, Amita Malik and Sanjay Kumar have reviewed the routing protocols and its issues for WMSN. This paper presents various existing routing strategies in WMSNs with their applications and limitations which lead to open research issues, detailed classification and comparison [5].

Almalkawi, Zapata and Karaki has proposed a cross layer based clustered multipath routing with QoS aware scheduling. It is based on clustered multipath routing protocol and adaptive QoS aware scheduling for different traffic classes. He has exploited hierarchical structure of powerful cluster heads and the optimized multiple path along with the adaptive scheduling to support reliable, high throughput and energy efficient multimedia transmission in WMSN [6].

\section{METHODOLOGY}

WMSN network is created with audio, video and temperature sensors. This network is heterogeneous in nature. Network simulator 2 is used to develop and simulate the performance of the FCAXL routing protocol. Throughput, jitter and packet delivery ratio are used as the performance measures to evaluate the performance. 
Network Parameters to simulate Routing Mechanism are set as shown in Table 1. These sensor nodes are embedded with data packets according to its type. The main aim of the design is to increase the network lifetime and to optimize the QoS parameters. Hence clustering in WMSN is challenging task. Clustering is done by assigning each node to particular cluster head. This cluster head selection is based on following parameters: Residual energy, distance between base station and sink, Memory and Distance between two clusters. This process is fully distributive. In the proposed method, fuzzy based clustering is used. The main objective of this method is to cluster the nodes in hierarchical way and to decrease the number of dead nodes.

Table 1. Network Parameters

\begin{tabular}{cc}
\hline Simulator & Network Simulator 2 \\
\hline Number of Nodes & Random \\
Topology & Grid \\
Interface Type & Phy/ WirelessPhy \\
MAC Type & 802.11 \\
Queue Type & Droptail /Priority Queue \\
Queue Length & 50 Packets \\
Antenna Type & Omni Antenna \\
Propagation Type & Two ray Ground \\
Routing Protocol & AODV \\
Transport Agent & UDP \\
Application Agent & CBR \\
Initial Energy & 100Joules \\
Simulation Time & 50seconds \\
\hline
\end{tabular}

Once the clustering is done Ant procedure is invoked to find accessible paths for all traffic which is created by the network to meet different QoS requirements. Here each sensor node waits for the announcement from the base station. Once the announcement is received from the base station then it looks for the route to the base station. If route is not available then FANTs are released in order to find the path with message to its neighbor. This message is sent to the base station through the intermediate nodes. In response, base station releases BANT message on reverse route. Thus intermediate nodes create the route via ant nodes. Base station continuously releases ants till the maximum ant release count reaches. Each node maintains maintenance timer, and checks for traffic load, if it reaches the threshold then it send MANT message with pheromone value. From the interface queue, MAC layer and Link layer packet service time is estimated.

$$
\text { T_PST }=\text { T_net }+\mathrm{T} \_q u e u e+T \_m a c+T \_t r a n s ;
$$

Sensor data is originated from various types of nodes. This data is audio, video and scalar type. Hence scheduling is done according to the priorities. Video data have highest priority while scalar data has lowest priority. Drop trail type queuing is used. For each $\mathrm{CH}$, classifier checks for the type of the packet and then sent to appropriate queue. Scheduler organizes these packets according to the level of priority.

When Node wants to communicate with sink it, it sends the information to the $\mathrm{CH}$. Then $\mathrm{CH}$ checks its routing table to find the appropriate shortest path based from the pheromone table. Once the route is discovered, data is sent to the sink. AntSenseNet algorithm is used to discover the path. Three phases are defined for this protocol: Forward ant Phase, Backward ant phase and route maintenance phase. In forward ant phase FANTS are generated to search the path towards the sink. FANTS carry the information about residual energy of the nodes and memory packet loss and queuing delay. These values are used as QoS metrics to discover the path.

When a forward ant reaches to the sink, it evaluates the suitable path and BANTs are generated in response. These BANTS carries the information of the corresponding FANTs about the intermediate nodes and are sent over the same path as FANT. In reverse journey pheromone values of intermediate $\mathrm{CHs}$ are updated. When BANT reaches the corresponding source node BANTS are thrashed and data is sent over the selected path.

Route maintenance phase deals with congestion and lost link problems. Here MANTs are generated which is set according to the traffic load on the link. In this, Hello messages are sent over the link periodically in order to check the connectivity of nodes. Once the route is discovered data packets are sent over the route following the maximum pheromone value. Multi path transmission is used in WMSN. 


\section{RESULTS}

In this paper performance of four different algorithms is evaluated for different scenario using NS2. For all simulations, 101 nodes are distributed in grid of $500 \mathrm{~m} \mathrm{X} \mathrm{500m} \mathrm{area.} \mathrm{Nodes} \mathrm{are} \mathrm{heterogeneous} \mathrm{in}$ nature i.e. audio, video and scalar nodes are used. Simulation parameters are set as shown in table no.1. All simulations are run for 50 Seconds. Fuzzy clustered ant based cross layered protocol is evaluated against AntSenseNet, Cross layered AntSenseNet, and Cross layered routing protocol on the basis of end to end delay, packet delivery ratio and throughput. Network is moderately loaded. Two types of data traffics were generated as scalar data and multimedia data. Multimedia traffic has higher priority than the scalar data traffic. Following parameters are evaluated:

Packet Delivery Ratio: PDR is the ratio of successfully delivered packet at the destination to the total number of packets sent from source to destination.

Throughput: Throughput is defined as the number of packets per second received at the sink node i.e. at destination.

From the Figure 1 it is observed that as PDR of Fuzzy clustered ant based XL WMSN lies between $65 \%$ to $98 \%$ which is higher than other three protocols. It is also is seen that PDR of ant based XL WMSN and XL WMSN is not less than $45 \%$. As the number of nodes increases, PDR also increases due to the decrease in the number of holes. As a result throughput increases. This can be observed from Figure2.

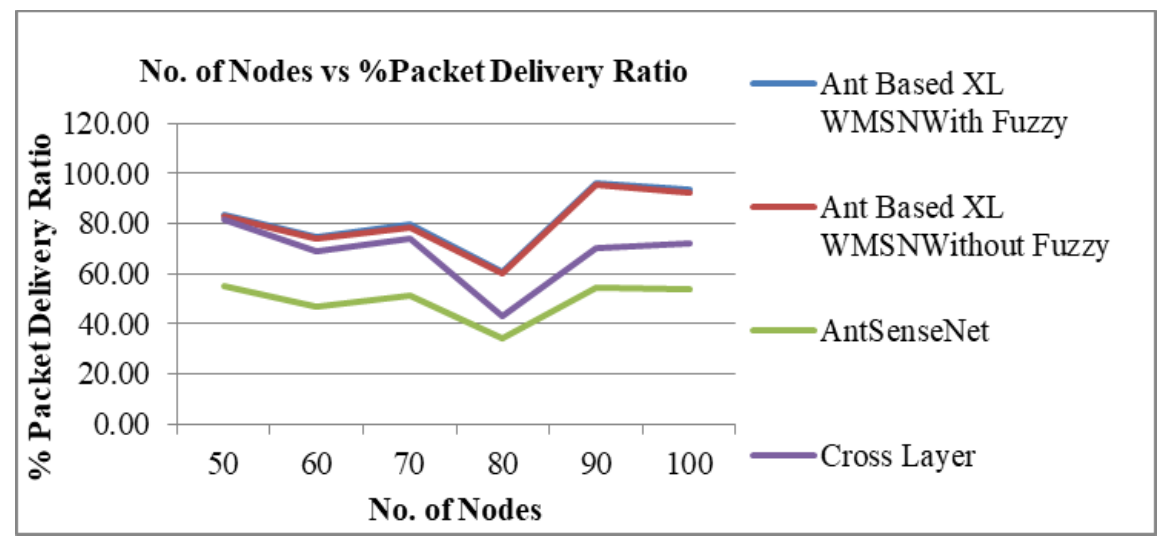

Figure 1. No. of Nodes vs Packet Delivery Ratio

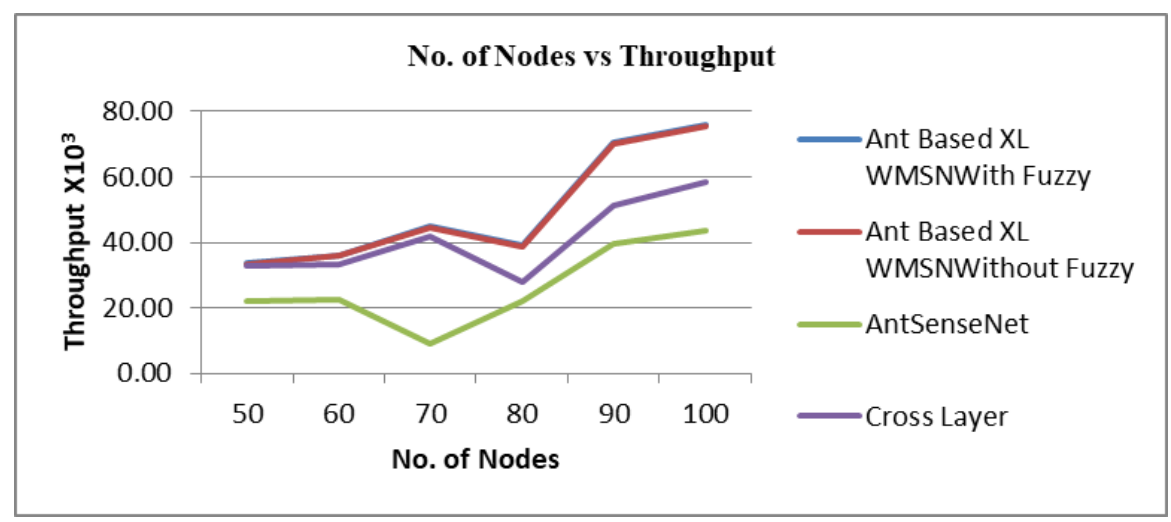

Figure 2. No. of Nodes vs Throughput

If packet size of the data is changed then FCAXL, Cross layer protocol and Ant based cross layer protocol has approximately same PDR. But still FCAXL WMSN performance is slightly improved as seen in Figure 3. 


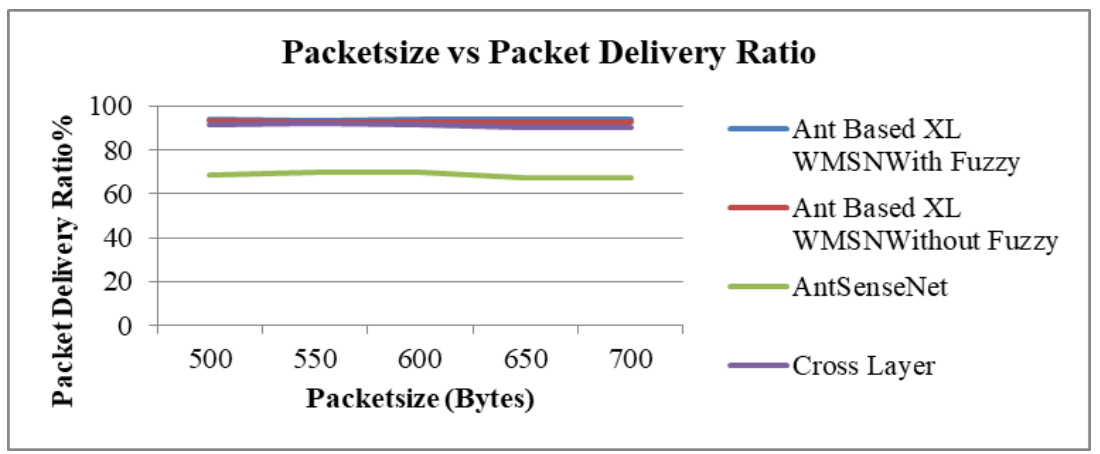

Figure 3. Packetsizevs packet Delivery Ratio

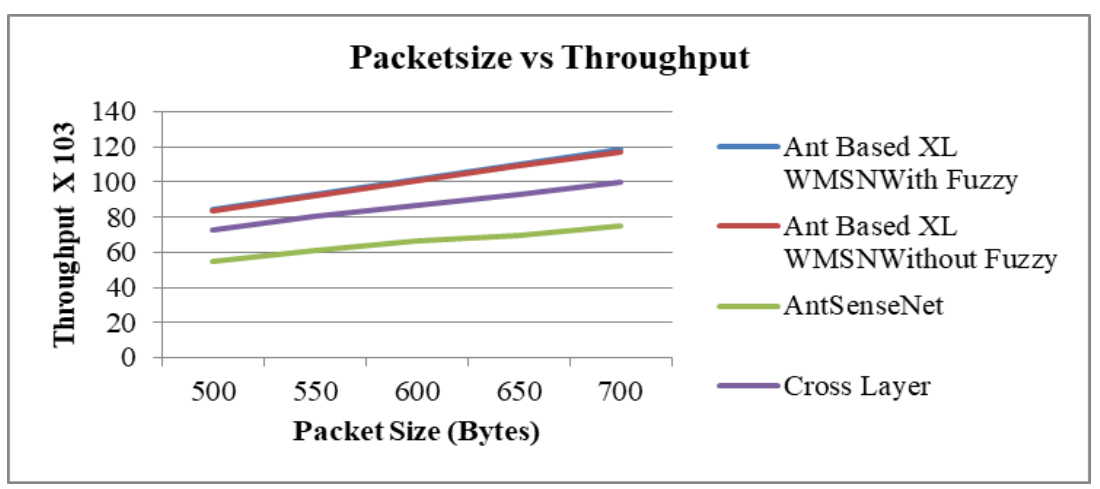

Figure 4. Packet size vs Throughput

End to end delay is thetime difference between the packet generation time and the time when it is received at the sink node.

As seen in Figure 5 if the number of nodes increases, end to end delay decreases and is much lesser than the other three algorithms for FCAXL protocol. This is because as the number of nodes increases distance between sink and source can be covered with minimum hops and as the multipath routing is employed dead link delay can be minimized. Also prioritrized scheduling algorithm is employed which results in decreses in delay.

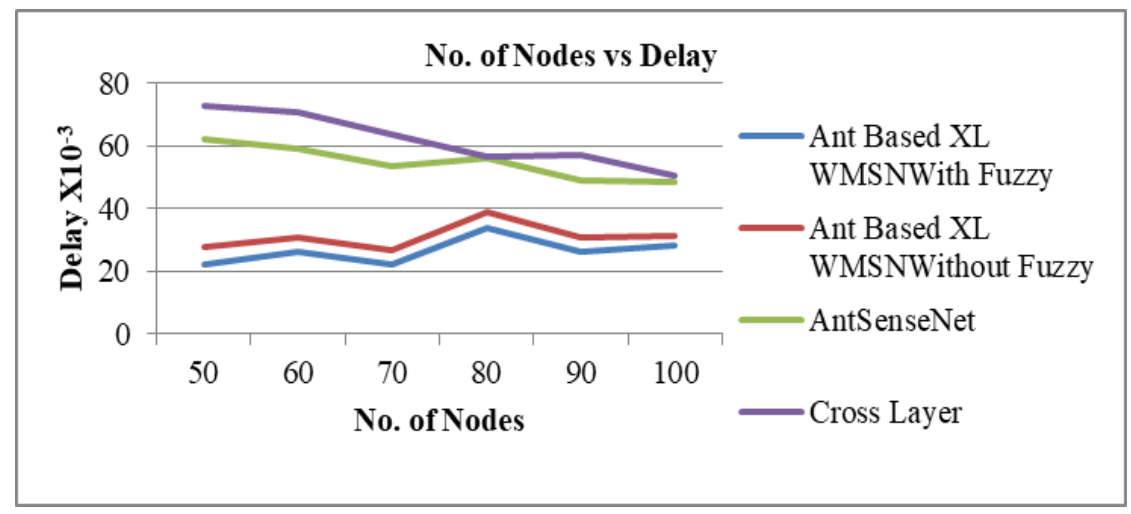

Figure 5. Number of Nodes vs Delay

Even if the packet size increases the delay remains minimum compared to other three algorithms as shown in Figure 6. 


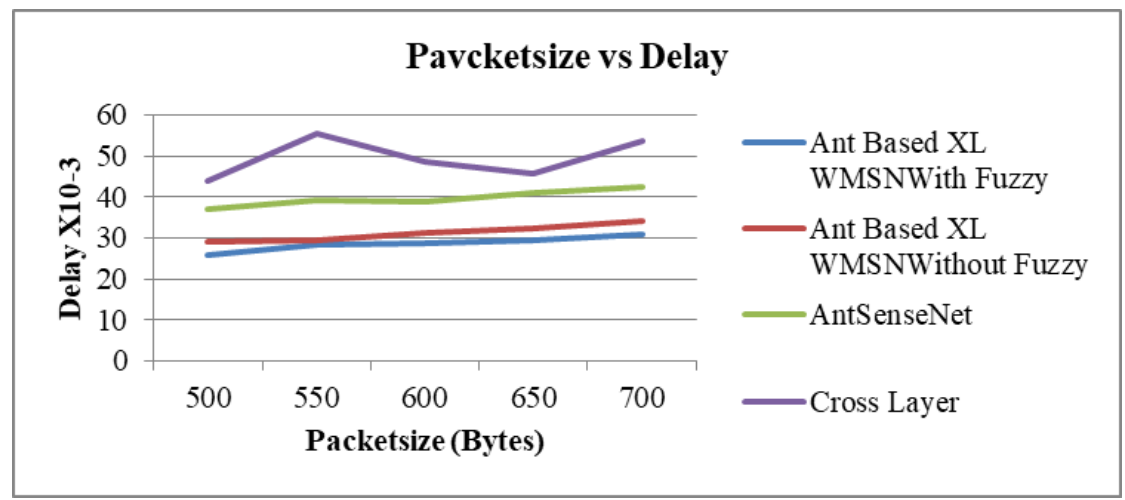

Figure 6. Packetsizevs Delay

\section{CONCLUSION}

WMSN is the network of heterogeneous sensors. It has many challenges such as QoS optimization, bandwidth, power, coverage and lifetime. In this paper, fuzzy clustered Ant based Cross layer protocol is proposed. Here due to fuzzy clustering dead nodes are minimized which increases throughput and in turn increases the network lifetime. Cross layering exploits interdependencies of the protocol layer which leads to increase in lifetime of the network and ant based routing algorithm helps to find shortest path which minimizes the end to end delay. Combined effect of all of these methods can be observed from the results shown. Thus from the simulation results it is observed that Fuzzy clustered Ant based cross layer routing protocol has better performance compared to other three algorithms in terms of end to end delay, throughput and packet delivery ratio.

\section{REFERENCES}

[1] Zara Hamid, Faisal Bashir and Jae Young Pyun, "Cross layer QoS routing protocol for multimedia communication in sensor networks", IEEE, pp498-502, 2012.

[2] Luis Cobo, Alejandro Quintero, Samuel Pierre, "Ant based routing for wireless multimedia sensor networks using multiple QoS metrics”, Elsevier. Computer Networks.54 pp 2991-3010, may 2010.

[3] M. Abazeed, K. Saleem, S. Zubair, N. Fisal, "CARMP: Cross layer based protocol for wireless sensor multimedia sensor network", Springer, pp1, 2011.

[4] G S M Vamsi, NehaChoubey, " A fuzzy based approach of energy efficient hierarchical clustering method in wireless sensor networks”, IJSR, pp 3001-3006, June 15.

[5] VikasBhandary, Amita Malik, Sanjay Kumar, "Routing I wireless multimedia sensor networks: A survey of existing protocols and open research challenges", Hindawi Publications, 2016.

[6] Islam T. Almalkawi, Manel Zapata, and Jamal N. Al-Karaki, "A cross layer based clustered multipath routing with QoS aware scheduling for wireless multimedia sensor networks", IJDSN, pp1-11, Volume 2012.

[7] Tamizharasi, A., Selvathai, J.J., KaviPriya, A., Maarlin, R., Harinetha, M., "Energy aware heuristic approach forcluster head selection in wireless sensor networks" Bulletin of Electrical Engineering and Informatics, Vol. 6 , Issue 1, 2017, pp. 70-75.

[8] Saini, R.K., Ritika, Vijay, S., "Data flow in wireless sensor network protocol stack by using bellman-ford routing algorithm", Bulletin of Electrical Engineering and Informatics, Vol. 6, Issue 1, 2017, pp. 81-87.

[9] Toor, A.S., Jain, A.K., "A survey on wireless network simulators", Bulletin of Electrical Engineering and Informatics, Vol. 6, Issue 1, 2017, pp. 62-69.

\section{BIOGRAPHIES OF AUTHORS}

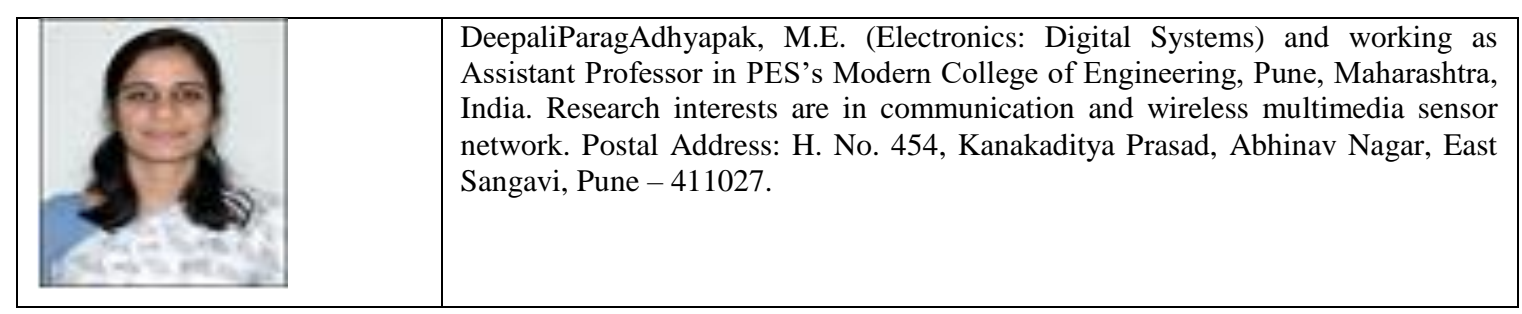




\begin{tabular}{|l|l|}
\hline & $\begin{array}{l}\text { SridharanBhavani is Ph.D. and working as Professor \& Head of Electronics \& } \\
\text { Communication Engineering Department in Karpagam Academy of Higher } \\
\text { Education, Coimbatore, Tamilnadu, India. Research interests are in image } \\
\text { processing, embedded systems, VLSI and wireless networks. Postal Address: } \\
\text { Pollachi Main Road, \& \& Ty Pass Road Junction Eachanari Post, Eachanari, } \\
\text { Coimbatore, Tamil Nadu 641021. }\end{array}$ \\
\hline & $\begin{array}{l}\text { Aparna Pradeep Laturkar, M.E.(Electronics) and working as Assistant Professor in } \\
\text { PES's Modern College of Engineering, Pune, Maharashtra, India. Research } \\
\text { interests are in communication and wireless sensor network. Postal Address: Flat } \\
\text { No. 301, AVALON, S, No. 18/1, 19, 20/3, Near Fire Brigade, Sun City Road, Off } \\
\text { Sinhgad Road, WadgaonBudruk, Pune - 411051. }\end{array}$ \\
\hline
\end{tabular}

